\title{
The Influence of Network Factors on Frequency Stability
}

DOI:

10.1109/TPWRS.2019.2958842

\section{Document Version}

Accepted author manuscript

Link to publication record in Manchester Research Explorer

\section{Citation for published version (APA):}

Fradley, J., Preece, R., \& Barnes, M. (2019). The Influence of Network Factors on Frequency Stability. IEEE Transactions on Power Systems, 35(4), 2826-2834. https://doi.org/10.1109/TPWRS.2019.2958842

\section{Published in:}

IEEE Transactions on Power Systems

\section{Citing this paper}

Please note that where the full-text provided on Manchester Research Explorer is the Author Accepted Manuscript or Proof version this may differ from the final Published version. If citing, it is advised that you check and use the publisher's definitive version.

\section{General rights}

Copyright and moral rights for the publications made accessible in the Research Explorer are retained by the authors and/or other copyright owners and it is a condition of accessing publications that users recognise and abide by the legal requirements associated with these rights.

\section{Takedown policy}

If you believe that this document breaches copyright please refer to the University of Manchester's Takedown Procedures [http://man.ac.uk/04Y6Bo] or contact uml.scholarlycommunications@manchester.ac.uk providing relevant details, so we can investigate your claim.

\section{OPEN ACCESS}




\title{
The Influence of Network Factors on Frequency Stability
}

\author{
J. Fradley, Student member, IEEE, R. Preece, Senior Member, IEEE and M. Barnes, Senior Member, \\ IEEE
}

\begin{abstract}
Retaining frequency stability is becoming increasingly challenging as the power system incorporates more non-synchronous generation. Assessing the frequency stability in a system has been predominantly completed by focusing on the quantity of connected synchronous kinetic energy in the system, or inertia. This traditionally was considered a function of generator construction - network factors typically were not considered. The research in this paper investigates how network topology, power flow, droop gain distribution, and inertia distribution all impact frequency stability. A generic four-area model has been created that allows discrete system setups. This research has shown that certain topologies lead to a more severe rate of change of frequency. A key finding is that the frequency drop is further increased when there is greater power flow into the area that experiences the disturbance. The extent to which the rate of change of frequency and frequency drop are influenced differently is emphasized, highlighting the need to procure different services depending on which metric is of primary significance at a specific location.
\end{abstract}

Index Terms - frequency containment, frequency stability, low inertia, ROCOF.

\section{INTRODUCTION}

$\mathrm{T}$ he need to decarbonize the power system in favor of renewable energy sources (RES) is leading to power system stability challenges. One pressing stability challenge is the ability to contain the system frequency following a disturbance. Inertial response from synchronous generators (SGs) currently limits the initial rate of change of frequency (ROCOF), providing time for the primary frequency response to activate and contain the frequency drop. It is important to contain the ROCOF and the maximum frequency drop to prevent rate of change relays and under frequency relays from operating and causing SGs to disconnect from the system, potentially leading to further cascading failures. Although there are moves to alter relay settings to cope with changes in operational norms (for example in the UK as given in [1]), the settings or the relay detection schemes may lead to SGs disconnecting during frequency containment events.

The main contributing factor for frequency stability is the level of synchronously connected kinetic energy within the system, commonly referred to as system inertia. The impacts of reduced system inertia are investigated in [2-4], and the

This work was supported by the EPSRC UK and Siemens through the EPSRC Centre for Doctoral Training in Power Networks (EP/L016141/1).

The authors are with the School of Electrical and Electronic Engineering, The University of Manchester, Manchester, M60 1QD, UK (e-mail: john.fradley@manchester.ac.uk; robin.preece@manchester.ac.uk). challenges posed for the system operators (SO) are evaluated in $[5,6]$. To ensure the system retains its frequency stability, the authors in $[7,8]$ attempt to determine the minimum level of inertia required. Numerous research articles deal with improving frequency stability by providing active power response from assets such as wind power plants (WPP) as in [9-11]. The option of using supplementary frequency control for power electronic interfaced RES is presented in [12, 13], and this can be expanded to use battery storage as in $[14,15]$. There are also studies investigating factors that influence frequency stability such as the influence of the governor control in [16-18] or where virtual inertia services should be located as in [19]. All of the methods reviewed were shown to improve frequency stability but most $\mathrm{SO}$ are not in the position of having numerous assets ready or capable of providing frequency response. The real time frequency variation around the system following a generator tripping is highlighted in [20], and shows that the system frequency around the system is not uniform. Due to this and the limited provisions of ancillary service capability, it is important to more fully understand the contributing factors that influence frequency stability at different points in a system to enable an SO to make more effective decisions to improve frequency stability.

A change in a certain network factor may have a more profound influence on either the frequency drop or the ROCOF. Determining which factors are of concern for each metric will allow the speed and quantity of the limited frequency response services to be better allocated in order to target a specific metric more strongly.

The main contributions of this paper are:

1) The identification and quantification of the variable impact that network factors have on the frequency drop and ROCOF in multi-area power systems.

2) An explanation of the mechanism through which power flow impacts frequency stability.

3) The creation of a measure of network topology that can be used to characterize different network connectivity and which is shown to have a strong relationship with the resultant system ROCOF.

The remainder of this paper is organized as follows. In Section II a review of frequency stability is provided and expanded in Section III to a system-wide analysis view point. The model used for analysis is introduced in Section IV and the results from the analysis are presented in Section V. Section VI provides the concluding remarks. 


\section{FREQUENCY STABILITY AND CONTAINMENT}

Following a large disturbance such as a generator disconnection, the additional power (or rather energy) to meet the load requirement is provided by the remaining generators connected to the system. The additional energy provided by the remaining generators in the moments after the disturbance comes from the kinetic energy that is intrinsically stored in the rotors, because of the rotating mass. This additional electrical power demand placed on the SG causes an increase in the electrical torque $\left(\tau_{e}\right)$ placed on the rotor given by (1) where $\omega_{r}$ is the machine's angular speed and $P_{e}$ is the electrical power. For a certain time period after the disturbance, the mechanical power input $\left(P_{m}\right)$ remains constant due to the slow acting ( $2 \mathrm{~s})$ governor control that operates the turbine fuel input valve. The resultant mechanical torque $\left(\tau_{m}\right)$ on the rotor remains approximately unchanged as given by (1). The net torque imbalance given by $\tau_{n e t}=\tau_{m}-\tau_{e}$, causes the rotor angular speed to alter as determined by the well-known swing equation in (2), where $H$ is the inertia constant and $S$ is the machine rating. The torque imbalance along with $H$ determines the rate of change of frequency, whereas the nadir is determined more by the mechanical response of the SG.

$$
\begin{gathered}
\tau_{e}=\frac{P_{e}}{\omega} ; \tau_{m}=\frac{P_{m}}{\omega} \\
\frac{d \omega}{d t}=\frac{\omega_{r}{ }^{2}}{2 H S} \tau_{n e t}
\end{gathered}
$$

Changes in electrical power around the system occur comparatively quickly, whereas, mechanical power changes are limited by the governor controls. Mechanical power cannot change instantly or in large quantities as this would cause excess stress to be placed on the generator's rotor. A visual depiction of how the torque imbalance on the rotor changes as time progresses following a system disturbance is given in Fig. 1. It displays the sudden initial large torque imbalance due to the pick-up followed by the slower acting torque balance restoring force of the primary response. The oscillations present in Fig. 1 are due to the use of a multimachine system used to generate the results. The stages of interest for frequency stability in this research are: initial torque imbalance (generator pick up), inertial response, and primary response.

\section{A. Initial Torque Imbalance (load pick up)}

The quantity of additional load that each generator initially picks up following a disturbance is important because it determines the initial torque imbalances on the machine. The amount of power injected by each SG can be determined through the solution of the system differential-algebraic equations (DAEs) that are provided in detail in [21, 22]. This has been extensively covered in literature and is fundamental to time domain simulation analysis. It is included here to fully describe what happens following a disturbance as it has implications for how different network factors affect frequency stability (as will be seen in Section V).

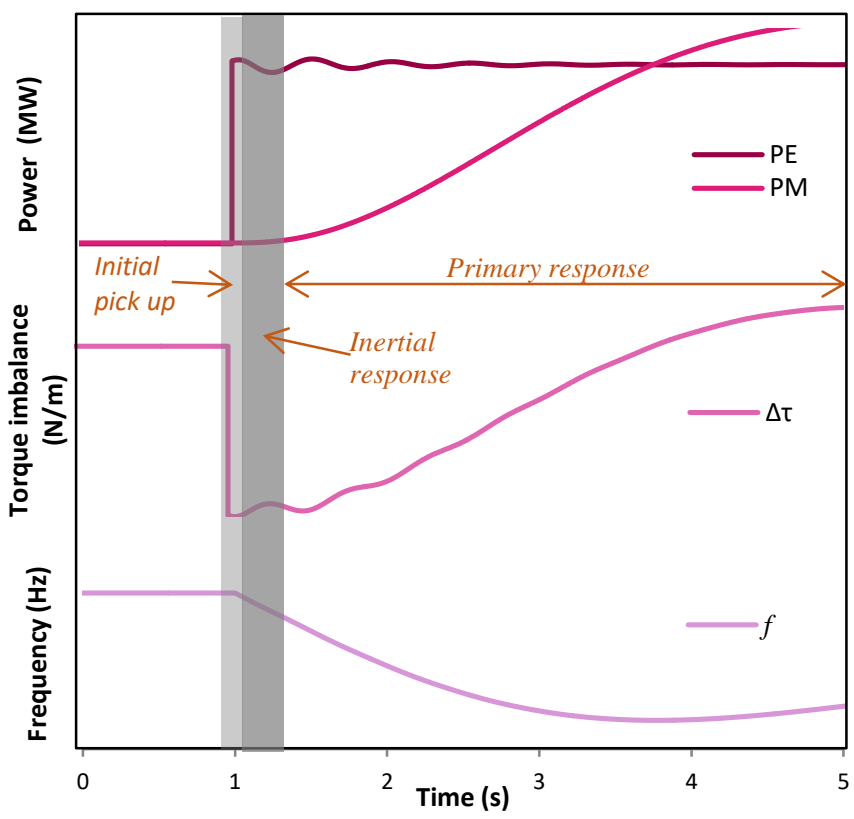

Fig. 1. SG power imbalances and mechanics following a disturbance

Starting with the simplified notation given by (3)-(5) which includes the stator algebraic equations in $\boldsymbol{h}$, the initial pick up is particularly concerned with solving the algebraic equations at the time of disturbance. The state variables in the differential equations in $\boldsymbol{f}$ do not change instantly and will be ignored for the timescales of interest. It should be noted that the symbol $\boldsymbol{f}$ is used within this section to describe the differential equations and is not related to frequency. Applying a partitioned solving method and using a Newton-Raphson (N$\mathrm{R})$ approach, the algebraic variables in $\boldsymbol{g}$ are updated by (6), where $\Delta P$ and $\Delta Q$ are the active and reactive power bus mismatches and $\boldsymbol{J}$ is the Jacobian matrix which determines the gradient change to a given algebraic variable.

$$
\begin{gathered}
\dot{\boldsymbol{x}}=\boldsymbol{f}(\boldsymbol{x}, \boldsymbol{u}) \\
\mathbf{0}=\boldsymbol{g}(\boldsymbol{x}, \boldsymbol{u}, \boldsymbol{I d}, \boldsymbol{I q}) \\
\boldsymbol{I} \boldsymbol{d}, \boldsymbol{I} \boldsymbol{q}=\boldsymbol{h}(\boldsymbol{x}, \boldsymbol{u}) \\
{\left[\begin{array}{c}
\theta \\
V \\
I d \\
I q
\end{array}\right]^{n+1}=\left[\begin{array}{c}
\theta \\
V \\
I d \\
I q
\end{array}\right]^{n}-[\boldsymbol{J}]^{-1}\left[\begin{array}{l}
\Delta P \\
\Delta Q
\end{array}\right]}
\end{gathered}
$$

The network node equations given in current form by (7) where $\boldsymbol{Y}$ is the admittance matrix and $\boldsymbol{I}$ is the node current injection matrix are used to determine the node mismatches. Using the power balance form, the active power $\left(P_{g i}\right)$ and reactive power $\left(Q_{g i}\right)$ injected from a generator into the network at bus $i$ is determined from the stator algebraic equations as in (8)-(9) where the subscript $i=1,2, \ldots, N$.

$$
\begin{gathered}
{[\mathbf{0}]=[\mathbf{Y}][\mathbf{V}]-[\boldsymbol{I}]} \\
P g_{i}=I d_{i} V_{i} \sin \left(\delta_{i}-\theta_{i}\right)+I q_{i} V_{i} \cos \left(\delta_{i}-\theta_{i}\right) \\
Q g_{i}=I d_{i} V_{i} \cos \left(\delta_{i}-\theta_{i}\right)-I q_{i} V_{i} \sin \left(\delta_{i}-\theta_{i}\right)
\end{gathered}
$$


Combining these into the active and reactive power balance equations for a generator bus produces the set in (10)-(11) [21] where the general bus values are denoted by the subscripts $i, n$ and the difference between two busses is denoted by the subscript in where $i=1,2, \ldots, N$. Completing the set of algebraic equations are the active and reactive power balance equations for a load bus as given by (12)-(13).

$$
\begin{aligned}
& 0=I d_{i} V_{i} \sin \left(\delta_{i}-\theta_{i}\right)+I q_{i} V_{i} \cos \left(\delta_{i}-\theta_{i}\right) \\
& -\sum_{n=1}^{N} V_{i} V_{n} Y_{i n} \cos \left(\theta_{i}-\theta_{n}-\theta_{i n}\right) \\
& 0=I d_{i} V_{i} \cos \left(\delta_{i}-\theta_{i}\right)-I q_{i} V_{i} \sin \left(\delta_{i}-\theta_{i}\right) \\
& -\sum_{n=1}^{N} V_{i} V_{n} Y \sin \left(\theta_{i}-\theta_{n}-\theta_{i n}\right) \\
& 0=-P_{l}+\sum_{n=1}^{N} V_{i} V_{n} Y_{i n} \cos \left(\theta_{i}-\theta_{n}-\theta_{i n}\right) \\
& 0=-Q_{l}+\sum_{n=1}^{N} V_{i} V_{n} Y_{i n} \sin \left(\theta_{i}-\theta_{n}-\theta_{\text {in }}\right)
\end{aligned}
$$

The Jacobian matrix consists of the partial derivatives for each active and reactive power equation with respect to the algebraic variables as given by (14) where it can be considered as four separate matrices when the stator variables are removed and form their own Jacobian.

$$
J=\left[\begin{array}{ll}
\frac{\partial P}{\partial \theta} & \frac{\partial P}{\partial V} \\
\frac{\partial Q}{\partial \theta} & \frac{\partial Q}{\partial V}
\end{array}\right]
$$

When the algebraic equations are updated $\left(\theta^{n+1}, V^{n+1}, I d^{n+1}, I q^{n+1}\right)$, they lead to new active and reactive power flows determined by (10)-(13), that in turn update the vectors $\Delta P$ and $\Delta Q$. These updated mismatch values drive the N-R solution until the mismatch errors are suitably reduced and then the differential equations can be solved. This is the basic format and well known method of the power flow solution. During this solution, the partial derivatives in $J$ determine the gradient or sensitivity at that specific operating point. Isolating the partial derivative for the active power of a generator bus in (15), it can be seen that the gradient is determined by the algebraic variables, $V, \theta, I d, I q$, state variable $\delta$, and network impedance $Y$.

$$
\begin{aligned}
\frac{\partial P_{i}}{\partial \theta_{i}}= & -I d_{i} V_{i} \cos \left(\delta_{i}-\theta_{i}\right)+I q_{i} V_{i} \sin \left(\delta_{i}-\theta_{i}\right) \\
& +\sum_{n=1}^{N} V_{i} V_{n} Y \sin \left(\theta_{i}-\theta_{n}-\theta_{i n}\right)
\end{aligned}
$$

These gradients determine how the algebraic variables change and subsequently how the power flow changes in (10)(13). The bus voltages are determined by the active and reactive power flows and the sensitivities of $d V / d P$ and $d V / d Q$ determine how the voltages change. The resultant voltage changes now impact the active power transfer and the power injection in the algebraic solution that ultimately determine the initial imbalance before the differential equations are solved.

The previous point highlights that if two identical generators are operating with the same active and reactive power output when a disturbance occurs at a symmetrical distance of $\boldsymbol{Y}$, then the initial pick up will be identical. If the two machines are operating at different power angles, or a machine is connected to a bus with a different voltage angle, then they would not pick up an identical amount of load. The machine that is operating at a larger power angle or that is connected to a bus with a greater voltage angle would subsequently experience a greater change in voltage drop. This resultant voltage drop leads to a reduced active power transfer from that SG. This explains the resultant quantity of load that the each generator picks up and, more importantly, it determines the electrical torque on the machine and therefore the rotor acceleration - which is of interest in this research.

\section{B. Inertial Response}

The inertial response is a natural response that occurs immediately and is predominantly determined from the wellknown swing equation given in per unit by (2). The analytical solution requires the solution of differential equations and the update of the machine states for each time step. The evolution of the machine's response is dependent on the torques imposed on the machine, machine damping, and the inertia of the machine. The inertia constant for a machine is the ratio of the stored angular kinetic energy of a generators rotor $\left(K_{E}\right)$ to the size or rating of the machine $(S)$ given by $K_{e} / S$. The time evolution of the frequency deviation is dependent on $H$ and the net torque $\left(\tau_{\text {net }}\right)$. To reduce the ROCOF requires either an increase in inertia or a reduction in the net torque.

\section{Primary Response}

The primary response in a typical SG is implemented into the turbine governing system and is a proportional control scheme, commonly known as droop control. The droop characteristic determines the change in power for a given change in machine speed given as (16) where $R$ is the droop coefficient and $P_{n}$ is the nominal power [23]. The primary response is contracted to start within a set timeframe by the system operator, but due to the physical constraints of the machine, it cannot start instantly nor have an excessive droop characteristic. As this response is proportional to the frequency deviation and delayed due to measurement, it has limited impact on the initial ROCOF and has a stronger impact on the frequency drop containment.

$$
\frac{\Delta \omega_{r}}{R \omega_{n}}=-\frac{\Delta P}{P_{n}}
$$

\section{FREQUENCY STABILITY CONTRIBUTING FACTORS}

Following a disturbance, certain factors affect the instantaneous changes (algebraic equations) and certain factors influence the time varying state changes (differential equations) as: 
- Algebraic equations: Network topology \& Power flow.

- Differential equations: Inertial response \& Primary response.

\section{A. Network Topology}

In order to understand how the network topology influences the frequency stability and response, it is beneficial to create a unique numerical measure for any given topology in order to be able to compare it against another topology. To distinguish each topology, the number of node connections, known as the average node degree (from graph theory) for each area and the electrical distance from the area where the disturbance occurs to every other area has been used in this research.

The average node degree is given by (17) where $\boldsymbol{k}$ is a network node and $\boldsymbol{N}$ is the number of connections into the node [24]. Using the system impedance matrix $\left(\boldsymbol{Z}_{\text {bus }}\right)$, the electrical distance as viewed from the disturbance area is given by (18) where $\boldsymbol{Z}$ is the total impedance and $\boldsymbol{Z}_{\boldsymbol{d} \boldsymbol{d}}$ is the self impedance in the area where the disturbance occurs. The subscript $\boldsymbol{j}$ refers to a network node or area in this case and $\boldsymbol{Z}_{\boldsymbol{d} j}$ refers to the connection between the area where the disturbance occurs and the area $\boldsymbol{j}$. Together they are used to create the overall topology measure $(\lambda)$ given by (19) that comprises of how connected and how distant the areas are. A high value of $\boldsymbol{\lambda}$ implies high impedance (distant areas) and low nodal connectivity. A low value of $\lambda$ is indicative of highly meshed low impedance networks.

$$
\begin{gathered}
k=1 / N \sum_{i=1}^{N} k_{i} \\
Z=\sum_{j=1}^{N-1}\left(Z_{d d}+Z_{j j}-2 Z_{d j}\right) \\
\lambda=Z / k
\end{gathered}
$$

The network topology strongly determines the system voltage angles and magnitudes that in turn determine the resultant generator pick up and post disturbance power flow. The topology also determines the rotor angle stability, where certain topologies cause greater rotor swings that are superimposed onto the frequency excursion. A weakly coupled system or an area that has a high electrical distance from the rest of the system will experience larger rotor angle swings, that when superimposed over the frequency excursion mean that the machine will experience periods where the frequency rate of change is higher than the center of inertia (or system) ROCOF as displayed in Fig. 2. This phenomenon can result in highly localized frequency behavior. As ROCOF techniques improve or the detection window reduces, these oscillations will need to be mitigated during the measurement process to prevent excessive or unwanted tripping.

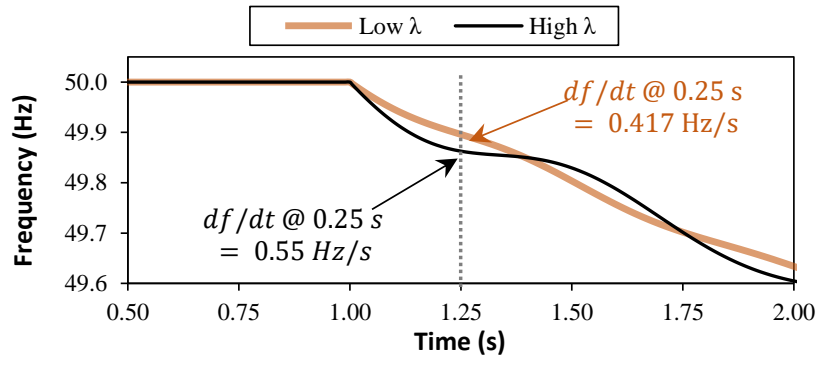

Fig. 2. Impact of topology on frequency response

\section{B. Power Flow}

The location and magnitude of the generation and the load determine the power flow around a network and subsequently determine the voltage magnitudes and angles. As outlined in Section II, the frequency stability and response of different synchronous machines will be different depending on the network operating points. The power flow into the area of the disturbance can be used as a quantity that indicates the voltage angle differences in relation to the area of disturbance. It can be extrapolated that the greater the power flow into the disturbance area, the greater the voltage angle differences will be with respect to the other areas due to the greater power transfer. As described in Section II.A, this resultant larger angle difference due to a greater power transfer into the disturbance area is expected to have a detrimental impact on frequency stability and lead to larger frequency drop and ROCOF.

\section{Inertial \& Primary Response Distribution}

Reducing inertia will decrease the frequency stability due to faster ROCOF. Increasing primary response droop gain will improve frequency stability as SG will actuate a greater response to frequency changes. When these factors are changed in different areas in relation to the disturbance area, they may lead to dissimilar frequency responses. Reducing inertia in the area where a disturbance occurs may lead to greater ROCOF than if the same inertia reduction occurs in another area. Increasing the primary response may be more beneficial in the disturbance area, or where the system experiences its worst frequency stability. Changing these factors in certain areas, could either provide system benefits or have limited impact on the overall frequency stability. The option may exist to target specific response types on specific areas depending on connected resources and operational conditions, particularly where the system is identified as weak with respect to frequency stability.

\section{Model AND ANALYSIS}

In order to assess the impact of various network conditions on frequency stability, a four area generic power system model has been developed and implemented in DIgSILENT PowerFactory 2017. The aim of the model is to allow an insightful view of how numerous network parameter distributions and topologies affect frequency stability. The model is designed to provide simple but precise topology 
changes without the need for laborious quantification as would occur in a larger system. For all simulation cases, the disconnection of a static generator occurs in Area4.

\section{A. Topology}

The model consists of four areas as depicted in Fig. 3. Each area can be connected using a simple set of connections or graphs where the transmission lines represent the edges.

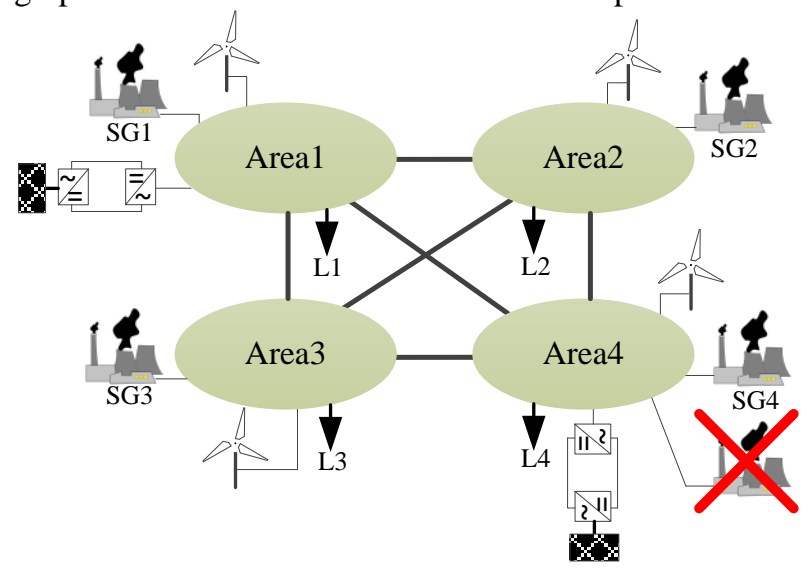

Fig. 3. Simulation test system. Disconnection occurs in Area4

The set of isomorphic topologies connecting all areas used in this research is given by Fig. 4 and the values of $Z, k$, and $\lambda$ for each graph are displayed in Table I. Other variants of connection that connect all areas exist but many of these are non-isomorphic graphs as they are rotated versions of the isomorphic graph. The different topologies (graphs) allow a logical investigation into how the coupling of the power system affects frequency stability. The different topologies are created by bringing lines in/out of service.

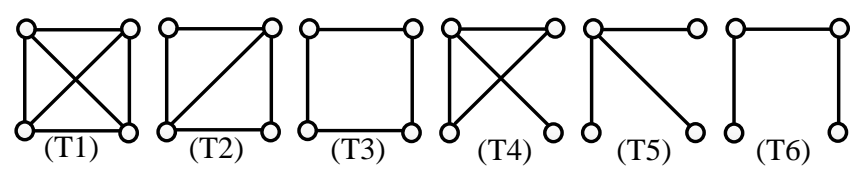

Fig. 4. Set of isomorphic topologies for four area system

TABLE I

TOPOLOGY MEASURES WITH RESPECT TO AREA4

\begin{tabular}{ccccccc}
\hline & $\mathrm{T} 1$ & $\mathrm{~T} 2$ & $\mathrm{~T} 3$ & $\mathrm{~T} 4$ & $\mathrm{~T} 5$ & $\mathrm{~T} 6$ \\
\hline $\mathrm{Z}$ & 4.7 & 7.5 & 8.4 & 11.6 & 14.1 & 22.6 \\
$\mathrm{k}$ & 3 & 2.75 & 2 & 2 & 1.5 & 1.5 \\
$\lambda_{\text {Area } 4}$ & 1.56 & 2.72 & 4.2 & 5.8 & 9.4 & 15.06 \\
\hline
\end{tabular}

\section{B. Generation}

Each area has an aggregated SG and an aggregated WPP. The SG model is a sixth order model that incorporates the electrical and mechanical torques on the rotor. The impact that different SG types, including their specific governor control, have on frequency stability is out of scope of the research at this stage. Previous research as in [25] has investigated the impact of different SG types on frequency stability and a future development of this analysis will be to incorporate different generation mixes. The WPPs are operated as constant power injection devices and do not provide any dynamic response in this research. Two VSC-HVDC in-feeds are incorporated into Area1 and Area4 and represent connection to a strong network; they also do not provide any dynamic response. The HVDC in-feeds are modelled using the average value Type-5 MMC model and incorporate vector control. All SGs have an automatic voltage regulator (AVR) of type IEEET1 and voltage setpoints of 1.01 p.u. A turbine governor is implemented in to each SG. As highlighted in [26] the intentional deadbands that are implemented into governor control loops in order to prevent excessive operation have an impact on the frequency response. Taking this into account, an augmented version of the IEEEG1 which has a deadband on the speed input is the WIESG1 and is used in this research to model turbine governor operation. A single type of governor is used at this stage in the research because all SG governor responses are proportional to the frequency deviation and they would respond in a similar manner. As the maximum ROCOF occurs immediately following the disturbance, the governor control has not had time to operate and this would be true for all variants. The deadband setting is $15 \mathrm{mHz}$ and the nominal droop gain is set at 20 p.u. (5\% droop). Variations in droop gain are detailed in Appendix Table III.

\section{Load Composition}

Each area has a constant power load to create worst case scenarios and represent the future trend in loads that are increasingly connected via power electronic converters. Different loading scenarios are set to alter the power flow around the network as defined in Appendix Table IV.

\section{Simulation Analysis}

A robust simulation methodology is used in which data is obtained from all cases involving six topologies, nine inertia distributions, eight load distributions, and nine droop gain distributions given in Appendix, and totaling 3888 simulation cases. For each case the maximum frequency drop and ROCOF that occurs in any area is recorded. Frequency drop is used as a measure instead of nadir so that both output variables increase to display worse frequency stability conditions. The frequency drop is the maximum difference between the nominal operating frequency and the nadir. The maximum ROCOF is detected over a $200 \mathrm{~ms}$ sliding window starting from the initiation of the disturbance in this research to capture the influence of the rotor angle stability on the frequency metrics. It is appreciated that different approaches and timeframes can be used to record the ROCOF but as yet, no standard approach has been agreed.

\section{RESUlts OF System ANALysis}

This section presents a selection of the key results obtained from analyzing the data set produced from undertaking all combinations of the possible network scenarios detailed in section IV. Due to space limitations, full details cannot be provided for all cases.

\section{A. Correlation}

Initial analysis of the results for all scenario combinations is undertaken using a Pearson multiple input linear regression correlation approach as outlined in [27]. The correlation is used to determine how a change in a certain variable affects frequency drop and ROCOF and specifically the degree of 
linear dependence between the variables and each metric. It highlights which parameters have the largest linear correlation to which metric. The variable $\sum$ PA4 represents power flow into Area4, $\lambda$ represents the network topology measure, HA1HA4 represent the inertia distribution in Area1-Area4, and RA1-RA4 represent the droop gain distribution in Area1Area4. The complete correlation results are displayed in Fig. 5.

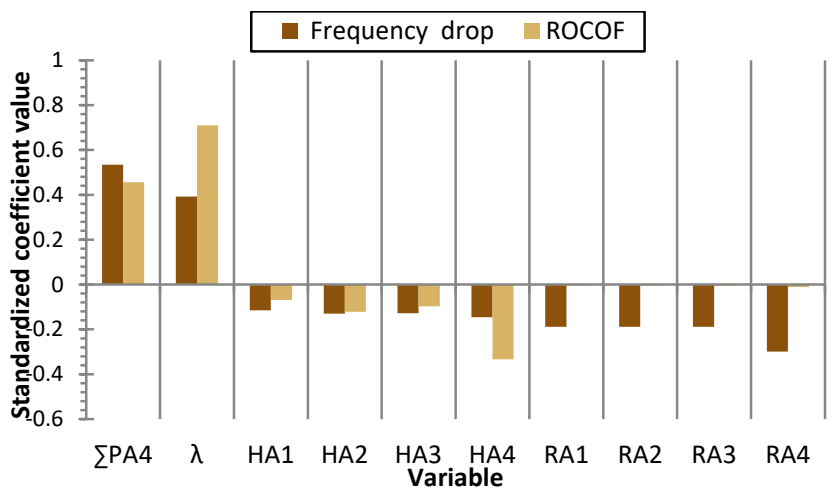

Fig. 5. System level factor correlation

The correlation confirms the knowledge that the primary response has limited impact on ROCOF because it has not had time to respond sufficiently in the moments following the disturbance. The results also display that an increase in Area4 inertia has the strongest effect on reducing ROCOF (again, as expected). A high value of $\lambda$ (i.e., a less connected system or one with larger electrical distances), is shown to have a strong positive correlation with both ROCOF and frequency drop. The results also confirm the theory that changes in power flows into Area4 lead to changes in frequency drop and ROCOF. These specific aspects will be examined in further detail in the remainder of this section.

\section{B. Impact of Topology and Power Flow}

The frequency drop and ROCOF for each topology is analyzed under the same load, inertia, and, primary response distribution. The maximum ROCOF observed in the network is plotted against the maximum frequency drop in Fig. 6. Picking this identical system setup for each topology shows that changing topology has a negligible impact on maximum frequency drop (y-axis of Fig. 6) but that there is a significant change in ROCOF of $0.132 \mathrm{~Hz} / \mathrm{s}$ between the best and worst ROCOF values (x-axis of Fig. 6). A closer examination of the cause reveals that topology T6 (the fully radial topology) causes larger rotor angle oscillations that are superimposed onto the frequency drop as displayed in Fig. 7. The results display that a larger value of $\lambda$ causes a greater ROCOF to be measured; however, there is negligible impact on the frequency drop as $\lambda$ increases.

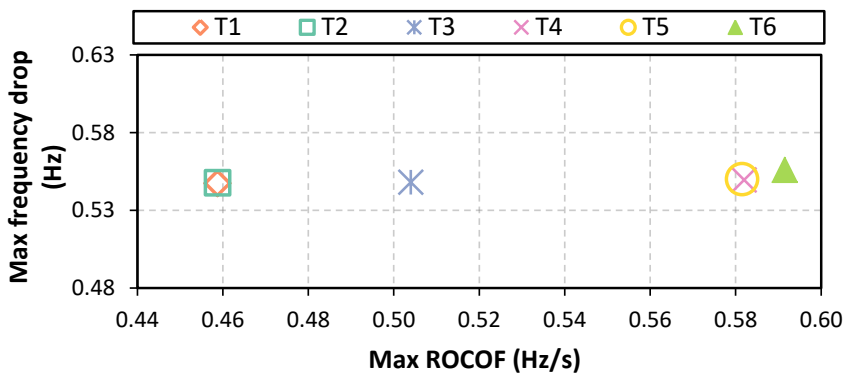

Fig. 6. Impact of topologies on ROCOF and frequency drop.

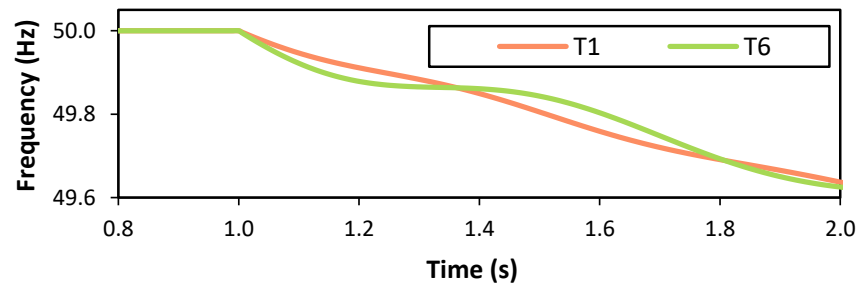

Fig. 7. Time series comparison between topology T1 and T6

Using the power flowing into Area4 as an indicator of the system operating point enables analysis of frequency drop and ROCOF as displayed in Fig. 8 and Fig. 9. The results display that for topology $\mathrm{T} 1$ there is a slight increase in both metrics when the power flow into Area4 increases. Whereas for topology T6, there is a significant increase in both metrics as the power flowing into Area4 increases. It was discussed in Section II.A how a larger voltage angle operating point with respect to Area4 will lead to greater voltage drops at those specific buses following system load disturbances, leading to less power being transferred. Considering topology T1, the post fault voltage drops for different power flows are shown in Fig. 10 where it can be seen that the greater the power flow into Area4, the higher the voltage drop following the sudden generator disconnection. Fig. 11 displays the voltage drops plotted against the pre-disturbance voltage angles with reference to Area4. It can be seen that a greater predisturbance voltage angle leads to greater voltage drops, indicating that the power transferred for a given change in angle during the updated power flow solution will be reduced. A time domain comparison between load distributions L7 and L8 using topology T1 is shown in Fig. 12, confirming the resultant increase in frequency drop. The frequency drop is further exacerbated when loading scenario L7 is combined with topology T6 as displayed in Fig. 13. This is due to even greater voltage angles between areas as this highly radial topology (with high $\lambda$ ) increases the impedance, and therefore the initial voltage angle differences, between areas.

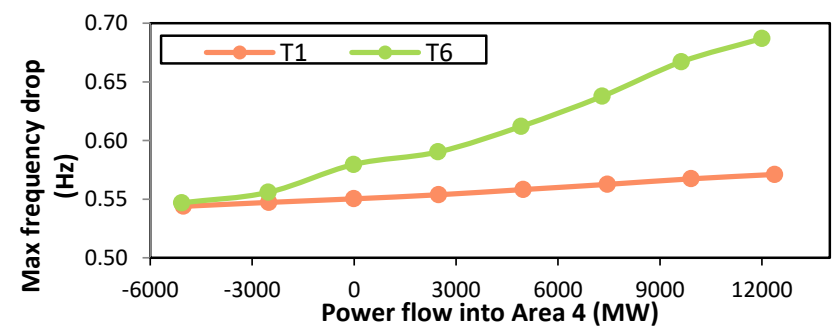

Fig. 8. Comparison of frequency drop for topology T1 and T6 


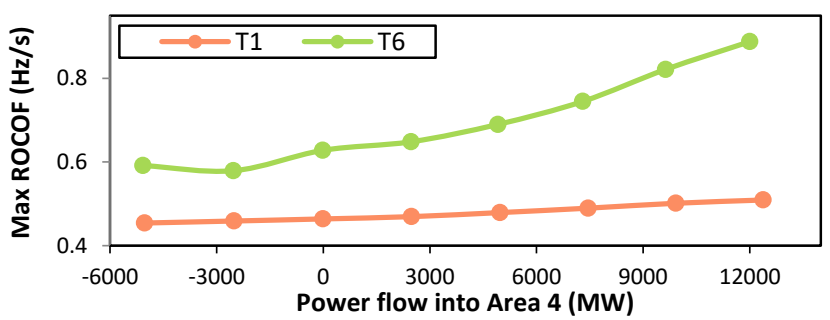

Fig. 9. Comparison of ROCOF for topology $\mathrm{T} 1$ and T6

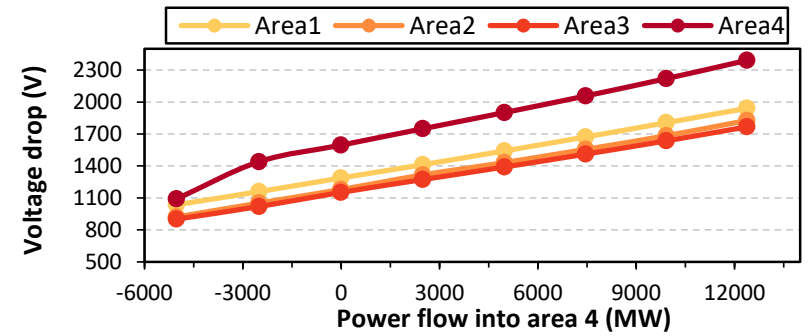

Fig. 10. Voltage drop for a pre disturbance power flow into Area4

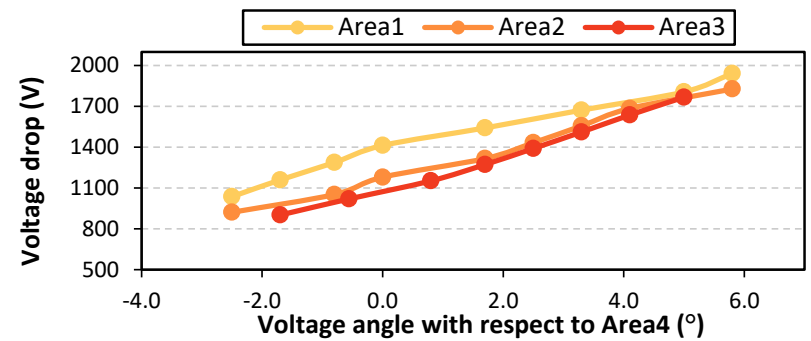

Fig. 11. Voltage drop vs pre fault voltage angle with respect to Area4

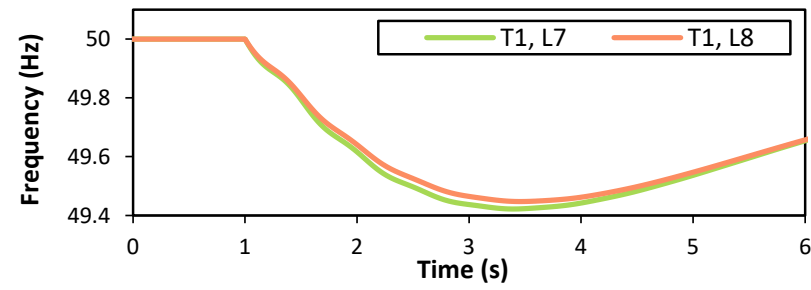

Fig. 12. Time series comparison for T1 with loading L7 and L8

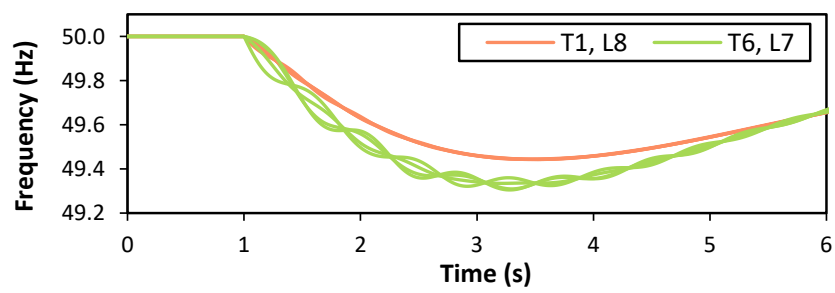

Fig. 13. Time series comparison for T1, L8 and T6, L7

\section{Impact of Inertia Distribution}

The impact of inertia reduction in a single specific area (without reducing it in other areas) is displayed in Fig. 14 and Fig. 15. This is equivalent to decommissioning or even temporarily disconnecting SGs from different areas of the network. The total system inertia before a reduction in a specific area is 120 GVA.s and the primary response remains unchanged in each area. The analysis uses topology $\mathrm{T} 1$ and loading L1 to create the base case conditions. Reducing the same amount of inertia in any area causes the same increase in frequency drop as shown in Fig. 14. Note that the y-axis here is scaled to be comparable to other plots included in this paper on frequency drop. It is acknowledged that the lines are essentially indistinguishable between different areas. In contrast, with respect to ROCOF, a reduction of inertia in Area4 results in a much greater change occurring. This is because the SG in Area4 will experience the greatest torque imbalance due to it being electrically closest to the disturbance. Changes in inertia will therefore have a greater relative impact with respect to the resultant SG acceleration. This set of results highlights that inertia support should be incorporated into areas that experience a reduction in inertia and that could be susceptible to large disturbances (e.g. areas with large interconnector in-feeds) while also having sensitive ROCOF relays operating.

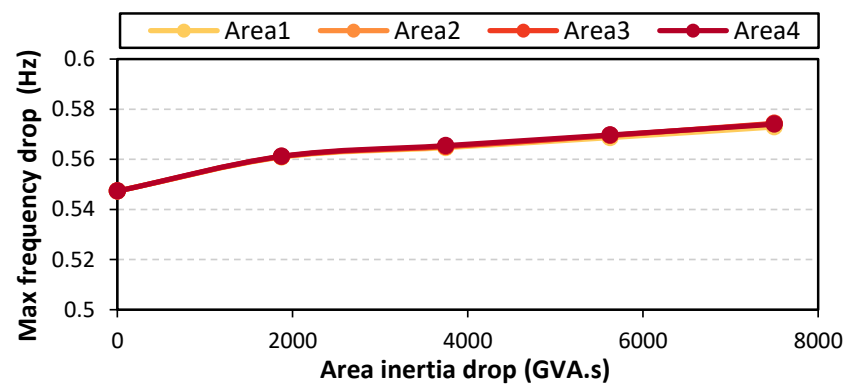

Fig. 14. Frequency drop for an inertia reduction in a specific area (N.B. lines are coincident as the y-axis is scaled to be comparable to other frequency drop plots).

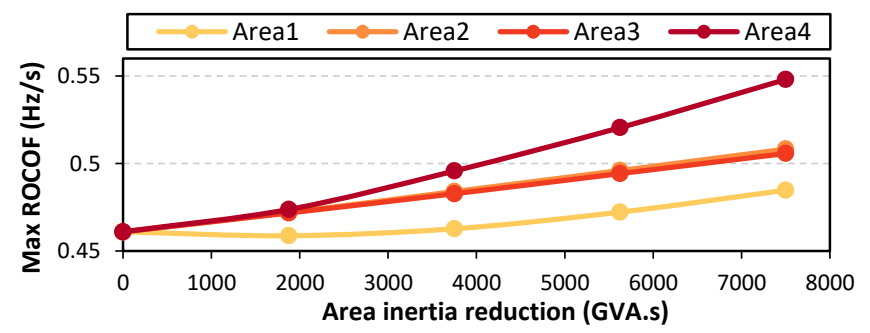

Fig. 15. ROCOF for an inertia reduction in a specific area

\section{Impact of Primary Response Distribution}

Simulation cases where the droop gain is increased in different areas one-at-a-time are analyzed in this subsection using topology $\mathrm{T} 1$ and loading $\mathrm{L} 1$ similar to the previous subsection. The increase of the droop gain in Area4 is shown to be more effective at reducing the nadir over cases where the same droop gain increase is applied to SGs in the nondisturbance area as displayed in Fig. 16. The droop gain increase does not reduce the severity of the initial maximum ROCOF seen in the system when the value is adjusted in any area as displayed in Fig. 17. This is due to the fact that the most severe ROCOF occurs immediately following the disturbance before the primary response has had time to detect and act. These results highlight that the primary response should be made more responsive (i.e. higher gain) in areas susceptible to large disturbances or in areas that have a high percentage of under frequency disconnection relays. 


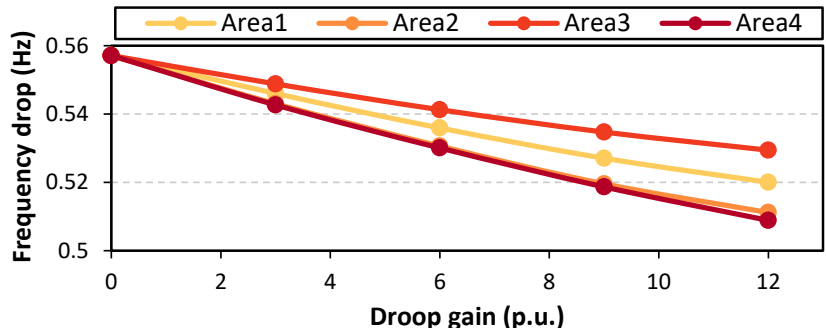

Fig. 16. Frequency drop for a droop gain increase in a specific area

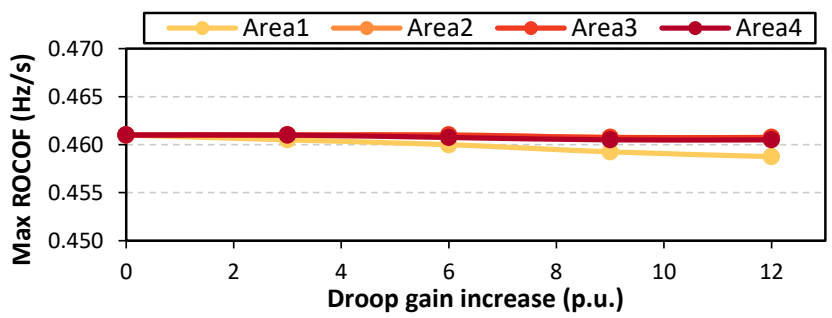

Fig. 17. ROCOF for a droop gain increase in a specific area (N.B. some lines are coincident as the $\mathrm{y}$-axis is scaled to be comparable to other plots ROCOF plots).

\section{E. Review of Factor Influence}

A general summary displaying the influence that an increase in a factor has on the frequency metrics is presented in Table III. An increase in a singular factor may only cause a negligible or slight increase in a certain metric. When there is an increase in multiple factors conjointly, such as the topology and power flow, the combination leads to a greater deterioration in both metrics.

TABLE II

SUMMARY OF FACTOR INFLUENCE ON FREQUENCY METRICS

\begin{tabular}{lcc}
\hline \multicolumn{1}{c}{ Factor (increase) } & Frequency drop & ROCOF \\
\hline Topology $(\lambda)$ & Negligible impact & Strong deterioration \\
Power flow (into disturbance area) & Slight deterioration & Slight deterioration \\
Topology and Power flow & Strong deterioration & Strong deterioration \\
Inertia reduction & Slight deterioration & Strong deterioration \\
Droop gain increase & Improvement & Negligible impact \\
\hline
\end{tabular}

From the factors presented, it can be concluded that the worst case operating scenario occurs when the is a high value of $\lambda$, a high power flow into the area of disturbance, and the droop and inertia distributions are focused outside of the area of disturbance. The impact these factors have on the frequency stability metrics can be used to inform the deployment of new forms of frequency response. It would be desirable to locate sources of synthetic or virtual inertia near to large SG in-feeds, instead of being located near load centers, to help alleviate initial torque imbalances following disturbances and reduce the ROCOF. More droop-based schemes could be deployed if an area had a high in-feed of power flow to limit the frequency drop experienced.

\section{CONCLUSION}

This research has explored frequency stability in greater depth with relation to the mechanisms and factors that govern the frequency drop and ROCOF in a power system. It has revealed how different network factors affect the time evolution of the frequency response and how specific parameters impact different frequency metrics. Network topology, described through the new metric $\lambda$ that combines connectivity and impedance, has been shown to influence both the frequency drop and the ROCOF. Frequency stability is further influenced by the power flow around the system in relation to the area where the disturbance occurs. The topology and power flow influence the operating points around the system which in turn determines the post fault torque imbalances (load pick up) for each SG. It has been shown that the power flow into the disturbance area can be used as an elementary indicator of subsequent frequency performance. The greater the power flow into the area of disturbance, the greater the frequency drop.

The location of the inertia reduction or the droop gain increase has also been shown to have a strong impact on the ROCOF and the frequency drop respectively. This work has shown the location-specific nature of these aspects and highlights that consideration should be given when deciding where to change these network factors.

Overall this research highlights that the frequency drop and ROCOF are influenced separately. It also displays that the factors governing them should be taken into consideration when allocating frequency response resources.

APPENDIX

TABLE III

DROOP GAIN DISTRIBUTION SCENARIOS

\begin{tabular}{lcccc}
\hline & AREA1 & AREA2 & AREA3 & AREA4 \\
\hline Droop dist. 1 $(\mathrm{pu})$ & 20 & 20 & 20 & 20 \\
Droop dist. 2 $(\mathrm{pu})$ & 25 & 20 & 20 & 20 \\
Droop dist. 3 $\mathrm{pu})$ & 33 & 20 & 20 & 20 \\
Droop dist. 4 $(\mathrm{pu})$ & 20 & 25 & 20 & 20 \\
Droop dist. 5 $(\mathrm{pu})$ & 20 & 33 & 20 & 20 \\
Droop dist. 6 $(\mathrm{pu})$ & 20 & 20 & 25 & 20 \\
Droop dist. 7 $(\mathrm{pu})$ & 20 & 20 & 33 & 20 \\
Droop dist. 8 $(\mathrm{pu})$ & 20 & 20 & 20 & 25 \\
Droop dist. 9 $(\mathrm{pu})$ & 20 & 20 & 20 & 33 \\
\hline
\end{tabular}

TABLE IV

DISPATCHED GENERATION AND LOADING DISTRIBUTION SCENARIOS

\begin{tabular}{lcccc}
\hline & AREA1 & AREA2 & AREA3 & AREA4 \\
\hline DisPATCH (MW) & 12500 & 12500 & 12500 & $\begin{array}{c}12500 \\
\text { (SLACK) }\end{array}$ \\
\hline Loading L 1 ( MW) & 15000 & 12500 & 12500 & 10000 \\
Loading L2 ( MW) & 15000 & 12500 & 10000 & 12500 \\
Loading L3 ( MW) & 15000 & 10000 & 10000 & 15000 \\
Loading L4 ( MW) & 12500 & 10000 & 10000 & 17500 \\
Loading L5 ( MW) & 10000 & 10000 & 10000 & 20000 \\
Loading L6 ( MW) & 7500 & 10000 & 10000 & 22500 \\
Loading L7 ( MW) & 7500 & 7500 & 10000 & 25000 \\
Loading L8 ( MW) & 15000 & 15000 & 12500 & 7500 \\
\hline
\end{tabular}

TABLE V

INERTIA DISTRIBUTION SCENARIOS

\begin{tabular}{lllll}
\hline & AREA1 & AREA2 & AREA3 & AREA4 \\
\hline Inertia dist. 1 (GVA.s) & 30000 & 30000 & 30000 & 30000 \\
Inertia dist. 2 (GVA.s) & 26250 & 30000 & 30000 & 30000 \\
Inertia dist. 3 (GVA.s) & 22500 & 30000 & 30000 & 30000 \\
Inertia dist. 4 (GVA.s) & 30000 & 26250 & 30000 & 30000 \\
Inertia dist. 5 (GVA.s) & 30000 & 22500 & 30000 & 30000 \\
Inertia dist. 6 (GVA.s) & 30000 & 30000 & 26250 & 30000 \\
Inertia dist. 7 (GVA.s) & 30000 & 30000 & 22500 & 30000 \\
Inertia dist. 8 (GVA.s) & 30000 & 30000 & 30000 & 26250 \\
Inertia dist. 9 (GVA.s) & 30000 & 30000 & 30000 & 22500 \\
\hline
\end{tabular}


Transmission line data: resistance: $0.015 \Omega / \mathrm{km}$, reactance: $0.15 \Omega / \mathrm{km}$, length $=50 \mathrm{~km}$

\section{REFERENCES}

[1] "National Electricity Transmission System Security and Quality of Supply Standard: Normal Infeed Loss Risk (GSR015)," Office of Gas and Electricity Markets. 2014, [Online]. Available: www.ofgem.gov.uk/publications-and-updates/security-and-qualitysupply-standard-sqss-gsr015-normal-infeed-loss-risk.

[2] A. Ulbig, T. S. Borsche, and G. Andersson, "Impact of Low Rotational Inertia on Power System Stability and Operation," IFAC, vol. 47, no. 3, pp. 7290-7297, 2014.

[3] C. Seneviratne and C. Ozansoy, "Frequency response due to a large generator loss with the increasing penetration of wind/PV generation - A literature review," Renewable and Sustainable Energy Reviews, vol. 57, pp. $659-668,2016$.

[4] P. Tielens and D. Van Hertem, "The relevance of inertia in power systems," Renewable and Sustainable Energy Reviews, vol. 55, pp. 9991009, 2016

[5] E. Ørum, M. Kuivaniemi, M. Laasonen, A. I. Bruseth, E. A. Jansson, et al, "Future system inertia," ENTSOE report, 2015.

[6] "Product Roadmap: For frequency response and reserve," National Grid Electricity Transmission plc .2017

[7] A. S. Ahmadyar, S. Riaz, G. Verbič, J. Riesz, and A. Chapman, "Assessment of minimum inertia requirement for system frequency stability," in Proc. IEEE International Conference on Power System Technology, 2016, pp. 1-6.

[8] H. Gu, R. Yan, and T. K. Saha, "Minimum Synchronous Inertia Requirement of Renewable Power Systems," IEEE Transactions on Power Systems, vol. 33, no. 2, pp. 1533-1543, 2018.

[9] Y. Phulpin, "Communication-Free Inertia and Frequency Control for Wind Generators Connected by an HVDC-Link," IEEE Transactions on Power Systems, vol. 27, no. 2, pp. 1136-1137, 2012.

[10] H. Ye, W. Pei, and Z. Qi, "Analytical Modeling of Inertial and Droop Responses From a Wind Farm for Short-Term Frequency Regulation in Power Systems," IEEE Transactions on Power Systems, vol. 31, no. 5, pp. 3414-3423, 2016

[11] J. V. d. Vyver, J. D. M. D. Kooning, B. Meersman, L. Vandevelde, and T. L. Vandoorn, "Droop Control as an Alternative Inertial Response Strategy for the Synthetic Inertia on Wind Turbines," IEEE Transactions on Power Systems, vol. 31, no. 2, pp. 1129-1138, 2016.

[12] J. Zhu, C. D. Booth, G. P. Adam, A. J. Roscoe, and C. G. Bright, "Inertia Emulation Control Strategy for VSC-HVDC Transmission Systems," IEEE Transactions on Power Systems, vol. 28, no. 2, pp. 1277-1287, 2013.

[13] A. Junyent-Ferr, Y. Pipelzadeh, and T. C. Green, "Blending HVDC-Link Energy Storage and Offshore Wind Turbine Inertia for Fast Frequency Response," IEEE Transactions on Sustainable Energy, vol. 6, pp. 10591066,2015

[14] D. M. Greenwood, K. Y. Lim, C. Patsios, P. F. Lyons, Y. S. Lim, and P. C. Taylor, "Frequency response services designed for energy storage," Applied Energy, vol. 203, pp. 115-127, 2017.

[15] H. Karbouj, Z. H. Rather, D. Flynn, and H. W. Qazi, "Non-synchronous fast frequency reserves in renewable energy integrated power systems: A critical review," International Journal of Electrical Power \& Energy Systems, vol. 106, pp. 488-501, 2019.

[16] F. Chengwei, W. Xiaoru, T. Yufei, and W. Wencheng, "Minimum frequency estimation of power system considering governor deadbands," IET Generation, Transmission \& Distribution, vol. 11, no. 15, pp. 3814$3822,2017$.

[17] G. Kou, P. Markham, S. Hadley, T. King, and Y. Liu, "Impact of Governor Deadband on Frequency Response of the U.S. Eastern Interconnection," IEEE Transactions on Smart Grid, vol. 7, no. 3, pp. 1368-1377, 2016

[18] I. Egido, F. Fernandez-Bernal, P. Centeno, and L. Rouco, "Maximum Frequency Deviation Calculation in Small Isolated Power Systems," IEEE Transactions on Power Systems, vol. 24, pp. 1731-1738, 2009.

[19] B. K. Poolla, D. Gross, and F. Dörfler, "Placement and Implementation of Grid-Forming and Grid-Following Virtual Inertia and Fast Frequency Response," IEEE Transactions on Power Systems, pp. 1-1, 2019.

[20] Z. Zhian, X. Chunchun, B. J. Billian, Z. Li, S. S. Tsai, R. W. Conners, et al., "Power system frequency monitoring network (FNET) implementation," IEEE Transactions on Power Systems, vol. 20, pp. 1914-1921, 2005.
[21] P. W. Sauer and M. A. Pai, Power System Dynamics and Stability. Prentice Hall, 1998.

[22] F. Milano, Power system modelling and scripting. Springer, 2010.

[23] P. Kundur, Power System Stability and Control. New Delhi: McGraw Hill, 1994.

[24] M. E. J. Newman, Networks: An introduction. Oxford University Press, 2010.

[25] E. Spahic, K. Frey, G. Beck, and V. Hild, "Inertia in the System and First Swing Frequency-Description and Mitigation Possibilities," in Proc. 2018 IEEE/PES Transmission and Distribution Conference and Exposition, Denver, USA, 2018, pp. 1-9.

[26] S. Mohajeryami, A. R. Neelakantan, I. N. Moghaddam, and Z. Salami, "Modeling of deadband function of governor model and its effect on frequency Response characteristics," In Proc. North American Power Symposium, Charlotte, USA, 2015, pp. 1-5.

[27] A. Edwards, An Introduction to Linear Regression and Correlation. USA: W. H. Freeman and Company, 1976.

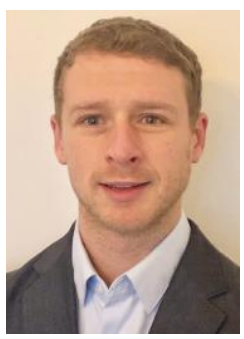

John Fradley received the B.Eng (Hons) degree in electrical and electronic engineering from the University of Staffordshire, U.K., in 2015. He is currently working towards the Ph.D. degree at The University of Manchester, U.K. His area of research is low inertia power systems.

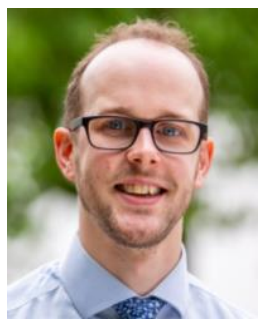

Robin Preece (M'13, SM'18) is a Senior Lecturer in Future Power Systems in the Department of Electrical and Electronic Engineering at The University of Manchester, where he has been an academic since July 2014. He previously received is BEng and $\mathrm{PhD}$ degrees from the same institution. His research is focused on the stability and operation of future power systems.

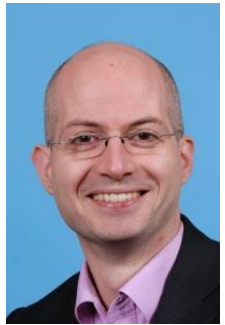

M. Barnes (M'96-SM'07) received the B.Eng. and $\mathrm{Ph} . \mathrm{D}$. degrees in power electronics and drives from the University of Warwick, Coventry, U.K., in 1993 and 1998, respectively. He is currently Professor of Power Electronics Systems at The University of Manchester. His research interests cover the field of power-electronics-enabled power systems. 